\title{
Recognizing Pinhole Parallax Error for Accurate Localization of Thyroid and Parathyroid Disease
}

\author{
Ismet Sarikaya $^{1}$, Zaina Al-Banna ${ }^{2}$, and Ali Baqer ${ }^{2}$ \\ ${ }^{I}$ Department of Nuclear Medicine, Kuwait University Faculty of Medicine, Safat, Kuwait; and ${ }^{2}$ Department of Nuclear Medicine, \\ Mubarak Al-Kabeer Hospital, Jabriya, Kuwait
}

\begin{abstract}
Parallax error is a pitfall of pinhole scintigraphy that causes mislocalization of the findings. It is important to notice this error on pinhole thyroid and parathyroid scintigraphy and obtain additional images with a parallel-hole collimator to accurately determine the upper and lower margins of large goiters and nodules and the location of ectopic thyroid tissue and parathyroid adenomas. In this teaching case report, we revisit pinhole parallax error, present pinhole and SPECT/CT images of a patient with a large, hyperactive thyroid nodule, and review the literature and potential solutions to this important problem.

Key Words: parallax error; pinhole collimator; thyroid scintigraphy; parathyroid scintigraphy; SPECT/CT
\end{abstract}

J Nucl Med Technol 2018; 46:288-289

DOI: $10.2967 /$ jnmt.118.207928

$\mathbf{U}$ se of a pinhole collimator is highly recommended for thyroid scintigraphy because it provides the only method of magnification, which is associated with better intrinsic resolution, contrast, and sensitivity than are obtainable with a parallel-hole collimator $(1,2)$. However, pinhole collimators can cause geometric distortion of objects with depth (3-5). The upper and lower margins of a large goiter or nodule, and the location of ectopic thyroid tissue and parathyroid adenomas, can be incorrect on routine pinhole thyroid and parathyroid scintigraphy. Pinhole parallax error was previously reported in a case of substernal goiter and another of parathyroid adenoma $(3,4)$. In this teaching case report, we present a case of pinhole parallax error in a patient with a large hyperactive thyroid nodule, and we briefly review the literature and discuss the potential solutions to this important problem.

\section{CASE REPORT}

A 24-y-old man with subclinical hyperthyroidism was referred to the Nuclear Medicine Department for a scinti-

\footnotetext{
Received Jan. 8, 2018; revision accepted Mar. 5, 2018.

For correspondence or reprints contact: Ismet Sarikaya, Department of Nuclear Medicine, Faculty of Medicine, Kuwait University, P.O. Box 24923, Safat, Kuwait 13110.

E-mail: isarikaya99@yahoo.com

Published online May 3, 2018.

COPYRIGHT (c) 2018 by the Society of Nuclear Medicine and Molecular Imaging.
}

graphic thyroid uptake study. Twenty minutes after intravenous administration of $185 \mathrm{MBq}(5 \mathrm{mCi})$ of ${ }^{99 \mathrm{~m} T c}$-pertechnetate, routine pinhole images of the thyroid gland were obtained in right anterior and left anterior oblique views using an anterior marker. The duration of the pinhole acquisition was 150 kilo counts for each view, and the matrix size was $256 \times 256$. The distance from the pinhole collimator to the patient was adjusted so that the gland occupied two thirds of the field of view. Thyroid scintigraphy was performed using a SoloMobile $\gamma$-camera (DDD-Diagnostic A/S) equipped with a pinhole collimator (3-mm insert). An additional SPECT/CT image of the neck and upper mediastinum was also obtained using a GE Healthcare Discovery 670 16-slice SPECT/CT camera. First, low-dose CT was performed for attenuation correction and anatomic localization. Afterward, SPECT images were obtained at $25 \mathrm{~s}$ per view and 60 views. Iterative reconstruction was used for image processing. The SPECT, CT, and SPECT/CT images were reviewed in the transaxial, sagittal, and coronal planes, and a maximum-intensity projection was reviewed as well.

The pinhole images showed a large, heterogeneous, hyperactive nodule in the left lobe and suppression of uptake in the right lobe (Fig. 1). The lower margin of the nodule was far above the suprasternal notch. However, the SPECT/CT images showed that the lower portion of the nodule was at the level of the suprasternal notch (Fig. 2).

\section{DISCUSSION}

The parallax error is caused by the combination of offcenter positioning of the pinhole in relation to the object and the inherent minification effect of the pinhole collimator with increasing distance (3). McKitrick et al. reported the parallax error in a patient with substernal goiter who was imaged using a pinhole collimator positioned at the usual thyroid bed between the thyroid cartilage and the suprasternal notch (3). On the resulting image, the substernal portion of the thyroid appeared as if it were within the normal thyroid bed. An additional image obtained with a rectilinear scanner showed that the lower margin of the goiter was substernal. To demonstrate this parallax phenomenon, McKitrick et al. also performed a study using a thyroid phantom. Serial images were obtained at increasing depths of the phantom, which was intentionally placed off center from the pinhole. As the pinhole-to-phantom distance 


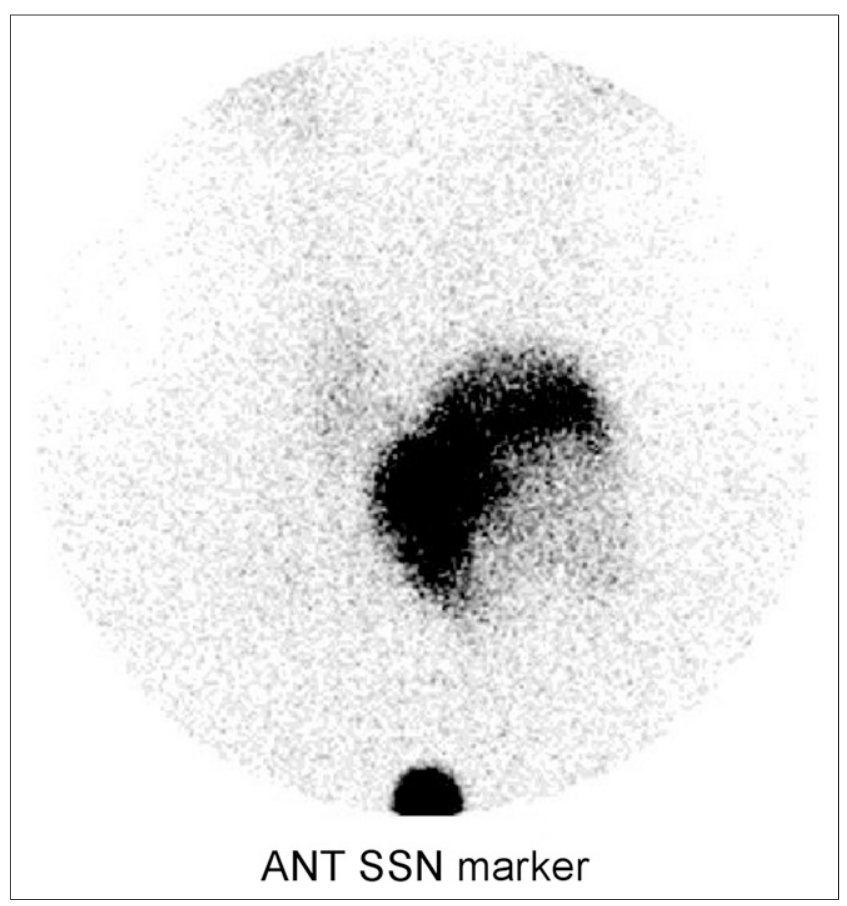

FIGURE 1. ${ }^{99 m}$ Tc-pertechnetate anterior (ANT) pinhole image of thyroid gland with marker at suprasternal notch (SSN) reveals large, hyperactive nodule in left lobe. Nodule shows cold areas laterally, likely because of degeneration or necrosis. Remainder of thyroid gland is suppressed, with only mild activity seen in right lobe. Lower margin of this nodule is far above suprasternal notch.

was increased, the thyroid image was "pulled up" to the normal location.

In another report, parathyroid adenoma was wrongly localized by pinhole ${ }^{99 \mathrm{~m}} \mathrm{Tc}$-sestamibi scintigraphy to the anterior mediastinum near a lower pole of the thyroid gland (4). After a failed surgical attempt to resect the adenoma, the patient was reimaged with a parallel-hole collimator and SPECT, which showed that the adenoma was near the heart, anterior to the carina.

In our patient, pinhole imaging erroneously showed the lower margin of the thyroid nodule to be far above the suprasternal notch. SPECT/CT helped to correctly locate the lower margin of the nodule.

This error can be avoided by centering the pinhole over the large goiter or nodule (3). In addition, using a parallelhole collimator, a planar, SPECT, or SPECT/CT image of the thyroid and chest region (with markers at the thyroid cartilage and suprasternal notch) should also be obtained to better identify the upper and lower margins of the large

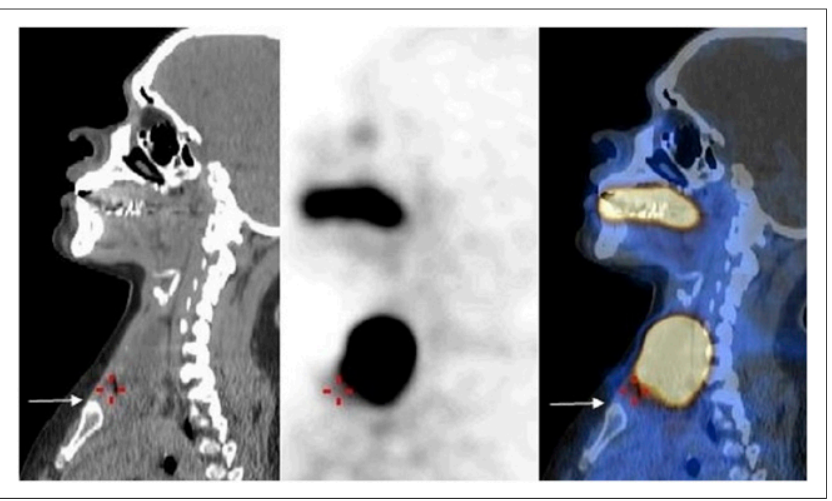

FIGURE 2. Selected ${ }^{99 m}$ Tc-pertechnetate sagittal CT, SPECT, and SPECT/CT images of thyroid gland show that lower margin of left thyroid nodule is at level of suprasternal notch. Arrow indicates upper margin of sternum.

goiter or nodule, determine whether it extends to the mediastinum, and locate ectopic thyroid tissue and parathyroid adenomas.

\section{CONCLUSION}

Pinhole scintigraphy is still commonly used to image the thyroid gland and is recommended for imaging parathyroid diseases. Hence, recognition of parallax error on pinhole thyroid and parathyroid scintigraphy is important, and if present, additional images should obtained with a parallelhole collimator to accurately determine the upper and lower margins of a large goiter or nodule and the location of ectopic thyroid tissue and parathyroid adenomas.

\section{DISCLOSURE}

No potential conflict of interest relevant to this article was reported.

\section{REFERENCES}

1. Ghanem MA, Elgazzar AH, Elsaid MM, Shehab F. Comparison of pinhole and high-resolution parallel-hole imaging for nodular thyroid disease. Clin Nucl Med. 2011;36:770-771.

2. Keyes JW Jr, Thrall JH, Carey JE. Technical considerations in vivo thyroid studies. Semin Nucl Med. 1978;8:43-57.

3. McKitrick WL, Park HM, Kosegi JE. Parallax error in pinhole thyroid scintigraphy: a critical consideration in the evaluation of substernal goiters. J Nucl Med. 1985;26:418-420.

4. Mavai R, Caride VJ. Pin-hole collimator, parallax, and the localization of mediastinal parathyroid adenoma. Clin Nucl Med. 1996;21:527-529.

5. Delbeke D, Coleman RE, Guiberteau MJ, et al.; Society of Nuclear Medicine (SNM). Procedure guideline for SPECT/CT imaging 1.0. J Nucl Med. 2006;47: $1227-1234$ 